\title{
Abnormal sub-pathways competitively regulated by IncRNAs contribute to postmenopausal osteoporosis
}

\author{
BING-GANG GUAN ${ }^{1}$ and XIAO-XI CAI ${ }^{2}$ \\ ${ }^{1}$ Spine Surgery, Tianjin Hospital, Tianjin 300211; \\ ${ }^{2}$ Department of Orthopedics, Huadong Hospital Affiliated to Fudan University, Shanghai 200040, P.R. China
}

Received June 18, 2018; Accepted February 4, 2019

DOI: $10.3892 /$ etm.2019.7326

\begin{abstract}
Abnormal sub-pathways competitively regulated by long non-coding RNAs (lncRNAs) for postmenopausal osteoporosis (PO) based on integration of lncRNA-mRNA expression data and pathway network topologies were investigated. Interesting lncRNA-mRNA pairs were selected by Pearson's correlation coefficient (PCC) algorithm on the basis of lncRNA-miRNA and miRNA-mRNA interactions and gene expression profiles. Then, lncRNAs in interesting pairs were embedded into pathway graphs as signature nodes by linking to their regulated-mRNAs, and lncRNA competitively regulated pathways (LCRPs) were gained for PO patients. Moreover, sub-pathways were detected dependent on the shortest distance similarity and the pathway topology. The abnormal sub-pathways were determined utilizing the Wallenius approximation methods through evaluating the statistical significance of sub-pathways. In total 75 interesting lncRNA-mRNA pairs (representing 17 lncRNAs and 74 mRNAs) were identified. Subsequently, 42 LCRPs were extracted from pathway graphs by signature lncRNA regulated mRNAs. Moreover, 14 abnormal sub-pathways with $\mathrm{P}<0.05$ were obtained between PO patients and controls, such as sub-pathways of PI3K-Akt signaling pathway and long-term potentiation. This finding may facilitate understanding the molecular mechanism of PO, and point a new direction to identify potential biomarkers for treatment and prevention of the disease.
\end{abstract}

\section{Introduction}

Postmenopausal osteoporosis (PO), primary type of osteoporosis, is regarded as a systemic skeletal disease characterized by compromised bone strength predisposing to an increased risk of fracture (1). Clinically, major manifestations of

Correspondence to: Dr Xiao-Xi Cai, Department of Orthopedics, Huadong Hospital Affiliated to Fudan University, 221 Yan'an West Road, Shanghai 200040, P.R. China

E-mail: xiaoxicai2017@163.com

Key words: postmenopausal osteoporosis, lncRNA, mRNA, sub-pathway, topology
PO patients are low bone mass density (BMD), a fracture or even vertebral compression fracture occurring during routine daily activities without a specific fall or injury (2). In consequence, PO imposes a great burden on life of both individuals and their families. Currently, usual treatments for postmenopausal women with osteoporosis mainly include vitamin D, estrogens, bisphosphonates, and calcitonin $(3,4)$. Thus, an effective approach to overcome these limitations is an urgent need and a huge challenge for PO related research.

Simultaneously, previous studies have demonstrated that low BMD is the optimal predictor of fracture risk in asymptomatic PO $(5,6)$. Hence, a comprehensive understanding and explanation for molecular mechanism underlying the progression of BMD reduction is critical for PO patients. Increasing proofs showed that long non-coding RNAs (lncRNAs) are able to competitively adjust messenger RNAs (mRNAs) expression levels via sharing common microRNA (miRNA) binding sites with mRNAs $(7,8)$. In addition, identifying lncRNA competitively regulated pathways (LCRPs) give potential for uncovering molecular mechanism and exploring functions of lncRNAs in complicated human disease (8).

Pathway structure information helps to understand the physiology of diseases and the functions of the biological system (9). Moreover, Li et al suggested that key local sub-regions, rather than complete pathways, could subtly explain the etiology of diseases $(10,11)$. This finding revealed that more attention should be focused on sub-pathways rather than entire pathways (12). Therefore, in this study, we aimed to identify abnormal sub-pathways competitively regulated by lncRNAs through integrating lncRNA-mRNA expression profiles and pathway topologies for PO patients. The results might provide powerful evidence and give insights for uncovering the potential molecular mechanism underlying PO.

\section{Materials and methods}

Using lncRNA-mRNA expression data, the inference of abnormal sub-pathways between PO patients and normal samples consisted of four steps. The first step was to construct interesting lncRNA-mRNA pairs by Pearson's correlation coefficient (PCC) algorithm. Secondly, LCRPs were gained for PO patients. Thirdly, sub-pathways were detected dependent on the shortest distance similarity and the LCRP network topology. Ultimately, abnormal sub-pathways were determined 
by the Wallenius approximation methods via evaluating the statistical significance of sub-pathways.

Recruitment of IncRNA-mRNA expression data. The interactions between IncRNAs and mRNAs were built by media of miRNAs. Thus the first part was to download the known IncRNA-miRNA and miRNA-mRNA interactions from the experimentally validated small non-coding RNAs target Base (starBase) and miRecords, respectively. Here, the straBase (http://starbase.sysu.edu.cn/) offers a comprehensive exploration for IncRNA-miRNA interactions curated from published studies (13). While the miRecords (http://mirecords.umn. edu/miRecords) are considered as an integrated resource not only for experimental miRNA-target interactions but also for target prediction programs (14). Furthermore, the IncRNA-mRNA competitively regulated relationships were defined by meeting to the two conditions concurrently: i) hypergeometric test (15) of shared miRNAs satisfying the criterion $\mathrm{P}<0.05$; and ii) Jaccard coefficient (16) of shared miRNAs ranking in top 20\%. Consequently, 7,693 interactions (covering 835 lncRNAs and 1,749 mRNAs) were retained, termed with background IncRNA-mRNA interactions for PO patients.

Gene expression profiles with accessing number GSE56815 for PO patients were acquired from Gene Expression Omnibus (GEO, https://www.ncbi.nlm.nih.gov/geo). The data were deposited on Affymetrix Human Genome U133A Array [HG-U133A], and comprised of $40 \mathrm{PO}$ samples and 40 control samples. In order to optimize the data regardless of the terms of quality and quantity, standard pretreatments and normalizations $(17,18)$ were conducted on GSE56815. Next, the preprocessed probes were converted into gene structures immediately. As a result, 12,437 genes were obtained from the dataset for PO patients. With an attempt to increase the correlations between background lncRNA-mRNA interactions and PO patients, 12,437 genes were mapped to these interactions, and only the intersected lncRNAs and mRNAs were reserved as lncRNA and mRNA data for PO in the subsequent analyses.

Construction of interesting lncRNA-mRNA pairs. Using the lncRNA and mRNA data, the co-expression probability for any pair of IncRNA-mRNA was assessed by the PCC algorithm. The PCC is a measure of the linear correlation between two variables, and its values $(r)$ range from +1 to -1 , where +1 is total positive linear correlation, 0 is no linear correlation, and -1 is total negative linear correlation (19). Subsequently, the Fisher's Z-transformation was applied to evaluate the statistical significance for $r$, which converts $r$ to the normally distributed variable $Z(20,21)$. The Fisher's $Z$-transformation of $r$ was computed by the following formula:

$$
Z=\frac{1}{2} \ln \frac{1+r}{1-r}
$$

When $Z$ goes from negative infinity to positive infinity, $\mathrm{P}$ distributes from 0 to 1 , and any value of $\mathrm{P}$ will uniquely be matched with a value of $Z$ and vice versa (21). If a pair of IncRNA-mRNA had a $\mathrm{P}<0.05$, it would be regarded as interesting lncRNA-mRNA pair of PO patients for further exploitation.
Identification of LCRPs. The KEGG pathway enrichment analysis was carried out on gene lists in pretreated GSE54400 (DAVID, https://david.ncifcrf.gov). Particularly, Fisher's exact test was employed to determine the statistical significance of pathways (22). The P-values were corrected by the false discovery rate (FDR) in Benjamini \& Hochberg method (23). Pathways with $\mathrm{P}<0.001$ were considered to be significant between PO patients and control samples. These significant pathways were converted into undirected graphs and kept the original pathway structural information using R packages (10). Then, lncRNAs in the interesting lncRNA-mRNA pairs were embedded into pathway graphs as nodes through linking to their regulated-mRNAs. As a consequence, LCRP network for PO was gained, including lncRNA-mRNA competitively regulated edges and lncRNA nodes. In addition, these lncRNA nodes were named as signature nodes in LCRP network.

Location of sub-pathways. During this step, sub-pathways for PO were located according to the shortest distance similarity combined with LCRP network topology. We chose any two of signature nodes randomly and computed the shortest path between them. If the amount of molecules between each signature pair was less than $n$ then they were integrated into one node. Simultaneously, if the node number in the molecule sets within pathway was more than $k$, they would be thought as sub-pathways competing and regulated via signature lncRNAs. Specifically, the factor $n$ controlled the intensity of regulated pathways, whereas $k$ parameter measured the size of this sub-pathway. In this study, we applied $k=8$ and $n=1$ as default parameters.

Investigation of abnormal sub-pathways. For purpose of evaluating whether the sub-pathways were competing and regulated by signature lncRNAs comparing random Wallenius approximation methods were implemented to test the statistical significance. Here, the Wallenius approximation methods is a generalization of the hypergeometric distribution where items are sampled with bias (24). Particularly, it was executed by BiasedUrn model in R package (25). In detail, a weight $(W)$ was computed for every sub-pathway, which indicated the intensity of competing regulation via signature lncRNAs associated with the sub-pathway.

$$
W=1-\log _{2}\left(\frac{G_{L}}{P_{G}}\right)
$$

$P_{G}$ was the number of mRNAs in this sub-pathway, and $G_{L}$ stood for the number of mRNAs competitively regulated by signature lncRNAs within this sub-pathway. Subsequently, supposing that $x$ was the number of interesting mRNAs in interesting lncRNA-mRNA pairs; $N$ referred to the amount of mRNAs in the gene expression data; $m_{l}$ was on behalf of the number of mRNAs associated with this sub-pathway; and $m_{2}$ represented the number of interesting mRNAs annotated into this sub-pathway, a corrected P-value was produced for the candidate sub-pathway as the following formula:

$$
P=F\left(x, m_{1}, m_{2}, N, W\right)
$$

Moreover, the cut-off for abnormal sub-pathways between PO and controls was $\mathrm{P}<0.01$. 
Table I. Interesting lncRNA-mRNA pairs with $\mathrm{P}<0.05$.

\begin{tabular}{|c|c|c|c|c|c|}
\hline \multicolumn{2}{|c|}{ Pairs } & \multirow[b]{2}{*}{$\mathrm{P}$-value } & \multicolumn{2}{|c|}{ Pairs } & \multirow[b]{2}{*}{ P-value } \\
\hline lncRNA & mRNA & & lncRNA & mRNA & \\
\hline DLEU2 & SEC 24A & 0.00000544 & CROCCP2 & PTEN & 0.0183 \\
\hline CROCCP2 & CUL5 & 0.000208 & TTTY15 & CACNG4 & 0.0188 \\
\hline JRK & FGFR2 & 0.000237 & TTTY15 & HMGA2 & 0.0191 \\
\hline CROCCP2 & KAT2B & 0.000317 & YLPM1 & EIF4G3 & 0.0203 \\
\hline DLEU2 & ACSL4 & 0.000458 & PVT1 & CCND1 & 0.0209 \\
\hline DLEU2 & DNAJC3 & 0.000522 & DLEU2 & PPP1CC & 0.0210 \\
\hline DLEU2 & STX7 & 0.000679 & LINC00312 & SMAD4 & 0.0217 \\
\hline LINC00342 & SOCS1 & 0.0013 & DLEU2 & LSM1 & 0.0224 \\
\hline YLPM1 & ITPR1 & 0.0015 & JRK & IGF1R & 0.0224 \\
\hline JRK & RARA & 0.0016 & DLEU2 & UTP6 & 0.0226 \\
\hline LINC00312 & RBL2 & 0.0016 & SEMA3B & CHEK1 & 0.0231 \\
\hline DLEU2 & AP3S1 & 0.0017 & DLEU2 & PRPF40A & 0.0239 \\
\hline UBXN8 & CDKN1B & 0.0018 & YLPM1 & EIF5B & 0.0251 \\
\hline DLEU2 & SPCS3 & 0.0020 & LINC00342 & MYCN & 0.0253 \\
\hline DLEU2 & KPNA1 & 0.0022 & MCF2L-AS1 & TERT & 0.0261 \\
\hline LINC00312 & RB1 & 0.0023 & DLEU2 & EIF2B1 & 0.0265 \\
\hline DLEU2 & PRKAA1 & 0.0027 & TTTY15 & GYS2 & 0.0272 \\
\hline DLEU2 & PAIP1 & 0.0031 & DLEU2 & PLA2G4A & 0.0274 \\
\hline TTTY15 & GEMIN7 & 0.0032 & SLC38A3 & PIK3R2 & 0.0278 \\
\hline MAP3K14 & CCND2 & 0.0032 & C11orf95 & $\mathrm{E} 2 \mathrm{~F} 1$ & 0.0293 \\
\hline DLEU2 & PPP3R1 & 0.0041 & YLPM1 & NOTCH2 & 0.0317 \\
\hline DLEU2 & PPP1R12A & 0.0051 & DLEU2 & GABARAPL2 & 0.0325 \\
\hline TTTY15 & AMT & 0.0083 & SEMA3B & FGF2 & 0.0331 \\
\hline DLEU2 & SEC11A & 0.0084 & LINC00342 & BMPR2 & 0.0342 \\
\hline DLEU2 & PPP2R5C & 0.0087 & YLPM1 & MDN1 & 0.0343 \\
\hline UBXN8 & KRAS & 0.0095 & SEC22B & ESR1 & 0.0350 \\
\hline DLEU2 & CUL2 & 0.0097 & JRK & RPS6KA1 & 0.0373 \\
\hline YLPM1 & ARHGDIA & 0.0115 & YLPM1 & ZYX & 0.0379 \\
\hline UBXN8 & NRAS & 0.0121 & JRK & EIF4EBP1 & 0.0392 \\
\hline MCF2L-AS1 & CDH1 & 0.0123 & YLPM1 & HSPA5 & 0.0432 \\
\hline DLEU2 & PPP3CA & 0.0128 & CROCCP2 & NOTCH2 & 0.0435 \\
\hline DLEU2 & CSNK1G3 & 0.0136 & DLEU2 & ARHGEF6 & 0.0446 \\
\hline JRK & GRIN2A & 0.0156 & DLEU2 & BMS1 & 0.0447 \\
\hline SEMA3B & IFNG & 0.0157 & LINC00663 & ZNF764 & 0.0464 \\
\hline UBXN8 & PMAIP1 & 0.0165 & TTTY15 & CACFD1 & 0.0476 \\
\hline CROCCP2 & SIRT1 & 0.0168 & TTTY15 & CCNB2 & 0.0481 \\
\hline LINC00663 & ITPKA & 0.0176 & TTTY15 & ATG4B & 0.0499 \\
\hline SPON1 & SMO & 0.0181 & & & \\
\hline
\end{tabular}

lncRNA, long non-coding RNA; mRNA, messenger RNA.

\section{Results}

Interesting lncRNA-mRNA pairs. In this study, 7,693 background lncRNA-mRNA interactions involved in 835 lncRNAs and 1,749 mRNAs were recruited based on the starBase and miRecords when setting the thresholdings as $\mathrm{P}<0.05$ and top $20 \%$ Jaccard distribution. To make these interactions more correlated to $\mathrm{PO}$, we mapped
12,437 genes in pre-processed GSE56815 to them, and only took the intersections. As a result, a total of 44 lncRNAs and 1,498 mRNAs which had intersections with all genes were gained to construct random lncRNA-mRNA pairs. An $r$ value was assigned to each lncRNA-mRNA pair using the PCC method, and then a P-value was calculated according to the Fisher's $Z$-transformation test. The cut-off for interesting IncRNA-mRNA pairs between PO patients 
Table II. LCRPs with $\mathrm{P}<0.001$.

\begin{tabular}{|c|c|c|c|}
\hline Pathway ID & Pathway & P-value & $\begin{array}{l}\text { Number of matched } \\
\text { lncRNA-mRNA pairs }\end{array}$ \\
\hline hsa04151 & PI3K-Akt signaling pathway & $2.18 \mathrm{E}-09$ & 15 \\
\hline hsa04110 & Cell cycle & $2.07 \mathrm{E}-08$ & 16 \\
\hline hsa04722 & Neurotrophin signaling pathway & $2.91 \mathrm{E}-07$ & 13 \\
\hline hsa04010 & MAPK signaling pathway & $3.35 \mathrm{E}-07$ & 11 \\
\hline hsa04720 & Long-term potentiation & $3.70 \mathrm{E}-07$ & 9 \\
\hline hsa04810 & Regulation of actin cytoskeleton & $7.25 \mathrm{E}-07$ & 9 \\
\hline hsa05218 & Melanoma & $2.00 \mathrm{E}-06$ & 10 \\
\hline hsa05219 & Bladder cancer & $2.18 \mathrm{E}-06$ & 5 \\
\hline hsa05200 & Pathways in cancer & $2.24 \mathrm{E}-06$ & 9 \\
\hline hsa05215 & Prostate cancer & 4.69E-06 & 10 \\
\hline hsa05212 & Pancreatic cancer & $4.70 \mathrm{E}-06$ & 7 \\
\hline hsa05203 & Viral carcinogenesis & $7.68 \mathrm{E}-06$ & 6 \\
\hline hsa05213 & Endometrial cancer & $1.44 \mathrm{E}-05$ & 6 \\
\hline hsa05161 & Hepatitis B & $1.78 \mathrm{E}-05$ & 7 \\
\hline hsa04114 & Oocyte meiosis & $1.98 \mathrm{E}-05$ & 9 \\
\hline hsa05223 & Non-small cell lung cancer & $2.22 \mathrm{E}-05$ & 10 \\
\hline hsa05221 & Acute myeloid leukemia & $2.46 \mathrm{E}-05$ & 6 \\
\hline hsa04370 & VEGF signaling pathway & $3.64 \mathrm{E}-05$ & 6 \\
\hline hsa05214 & Glioma & $6.77 \mathrm{E}-05$ & 9 \\
\hline hsa04910 & Insulin signaling pathway & $9.25 \mathrm{E}-05$ & 9 \\
\hline hsa05216 & Thyroid cancer & $2.10 \mathrm{E}-04$ & 4 \\
\hline hsa05222 & Small cell lung cancer & $2.51 \mathrm{E}-04$ & 5 \\
\hline hsa04150 & mTOR signaling pathway & $3.72 \mathrm{E}-04$ & 9 \\
\hline hsa04730 & Long-term depression & $3.72 \mathrm{E}-04$ & 6 \\
\hline hsa04066 & HIF-1 signaling pathway & $6.64 \mathrm{E}-04$ & 6 \\
\hline hsa05031 & Amphetamine addiction & $6.65 \mathrm{E}-04$ & 5 \\
\hline hsa04140 & Regulation of autophagy & $6.74 \mathrm{E}-04$ & 4 \\
\hline hsa05166 & HTLV-I infection & $6.99 \mathrm{E}-04$ & 9 \\
\hline hsa04662 & B cell receptor signaling pathway & 8.64E-04 & 5 \\
\hline hsa04660 & T cell receptor signaling pathway & $1.28 \mathrm{E}-03$ & 6 \\
\hline hsa04510 & Focal adhesion & $1.30 \mathrm{E}-03$ & 6 \\
\hline hsa03060 & Protein export & $1.65 \mathrm{E}-03$ & 3 \\
\hline hsa05220 & Chronic myeloid leukemia & $1.65 \mathrm{E}-03$ & 7 \\
\hline hsa04012 & ErbB signaling pathway & $2.02 \mathrm{E}-03$ & 3 \\
\hline hsa04914 & Progesterone-mediated oocyte maturation & $2.13 \mathrm{E}-03$ & 5 \\
\hline hsa04650 & Natural killer cell mediated cytotoxicity & $2.89 \mathrm{E}-03$ & 6 \\
\hline hsa05210 & Colorectal cancer & $3.82 \mathrm{E}-03$ & 4 \\
\hline hsa05211 & Renal cell carcinoma & $4.78 \mathrm{E}-03$ & 3 \\
\hline hsa04664 & Fc epsilon RI signaling pathway & $5.90 \mathrm{E}-03$ & 4 \\
\hline hsa04724 & Glutamatergic synapse & $6.96 \mathrm{E}-03$ & 5 \\
\hline hsa04115 & p53 signaling pathway & $8.01 \mathrm{E}-03$ & 5 \\
\hline hsa03013 & RNA transport & $8.48 \mathrm{E}-03$ & 6 \\
\hline
\end{tabular}

lncRNA, long non-coding RNA; mRNA, messenger RNA; LCRPs, IncRNA competitively regulated pathways.

and control samples was $\mathrm{P}<0.05$ in the present study. As displayed in Table I, in total 75 interesting lncRNA-mRNA pairs (representing 17 lncRNAs and 74 mRNAs) were identified. The most interesting pair between DLEU2 and SEC24A possessed the most significant $P$ of 5.44E-06, and the next two pairs were CROCCP2-CUL5 (P=2.08E-04) and JRK-FGFR2 (P=2.37E-04).

LRCPS. As described above, the KEGG pathway enrichment analysis was carried out on 12,437 genes in the gene 
Table III. Abnormal sub-pathways with $\mathrm{P}<0.01$.

\begin{tabular}{llcc}
\hline Sub-ID & \multicolumn{1}{c}{ Pathway } & Weight & P-value \\
\hline $4151 \_2$ & PI3K-Akt signaling pathway & 1.4854 & $<0.001$ \\
4720_1 & Long-term potentiation & 1.6374 & $<0.001$ \\
$5200 \_2$ & Pathways in cancer & 1.5475 & $<0.001$ \\
$5215 \_1$ & Prostate cancer & 1.6374 & $<0.001$ \\
4810_1 & Regulation of actin cytoskeleton & 1.5146 & $1.11 \mathrm{E}-16$ \\
$4110 \_2$ & Cell cycle & 1.6521 & $2.22 \mathrm{E}-16$ \\
$4010 \_1$ & MAPK signaling pathway & 1.2224 & $2.22 \mathrm{E}-15$ \\
$5223 \_1$ & Non-small cell lung cancer & 1.4150 & $8.88 \mathrm{E}-15$ \\
$5218 \_2$ & Melanoma & 1.7370 & $6.59 \mathrm{E}-14$ \\
$5166 \_2$ & HTLV-I infection & 1.0000 & $1.26 \mathrm{E}-13$ \\
$4114 \_2$ & Oocyte meiosis & 1.4854 & $2.63 \mathrm{E}-12$ \\
$5214 \_1$ & Glioma & 1.4854 & $2.63 \mathrm{E}-12$ \\
$4150 \_1$ & mTOR signaling pathway & 1.6781 & $7.00 \mathrm{E}-12$ \\
$4722 \_1$ & Neurotrophin signaling pathway & 1.6781 & $7.09 \mathrm{E}-12$
\end{tabular}

expression dataset. A total of 42 significant pathways with $\mathrm{P}<0.001$ were identified for PO patients (Table II). We found that $\mathrm{PI} 3 \mathrm{~K}-\mathrm{Akt}$ signaling pathway $(\mathrm{P}=2.18 \mathrm{E}-09)$, cell cycle $(\mathrm{P}=2.07 \mathrm{E}-08)$, and $\mathrm{p} 53$ signaling pathway $(\mathrm{P}=2.91 \mathrm{E}-07)$ were the three most significant pathways. In addition, each significant pathway was assigned to an ID, and the pathway stood by the end of four of the ID in the subsequent analysis. Next, 42 KEGG pathways were converted into undirected graphs that retained their original pathway structural information, and then the 17 lncRNAs were embedded into the graphs as signature nodes by linking to their regulated-mRNAs. Consequently, the total 42 significant pathways could be connected to IncRNAs by the regulation of mRNAs, and thus 42 LCRPs were explored for PO. In detail, 16 lncRNAmRNA interactions were enriched in the cell cycle. Besides, 11 lncRNAs regulated PI3K-Akt signaling pathway through 15 mRNAs since one lncRNA could regulate multiple mRNAs synchronously.

Abnormal sub-pathways. After identification of LCRPs for PO, their sub-pathways were explored through the integration of the shortest path similarity and LCRP network topology. The ID for sub-pathways were in accordance with the corresponding LCRPs, for example, the first sub-pathway for PI3K-Akt signaling pathway (ID: 4151) was described as 4151_1 and the second one was 4151-2. In fact, these subpathways mostly belonged to 14 of 42 LCRPs. Immediately, the Wallenius approximation methods was used to evaluate the statistical significance of sub-pathways and executed dependent on R package BiasedUrn. Consequently, a total of 14 abnormal sub-pathways were determined under the condition of $\mathrm{P}<0.01$, as shown in Table III.

In order to illustrate the correlations more directly and clearly, a sub-pathway network for signature nodes and abnormal sub-pathways was constructed and visualized by Cytoscope (Fig. 1). As one LCRP might have several sub-pathways, we selected the ID for corresponding LCRPs to represent its multiple sub-pathways in the sub-pathway network conveniently. The network comprised of 66 nodes and 129 edges, of which 14 were LCRPs, 14 signature lncRNAs and 38 mRNAs. Then, we calculated the topology centrality of this network using the degree index. Interestingly, the degree for YLPM1 and PI3K-Akt signaling pathway (ID: 4151) in both was 58, and followed Neurotrophin signaling pathway (ID: 4722, degree $=48$ ) and TTTY15 (degree $=47$ ). These nodes with high degree might play a more important role in the progression of PO patients, which also were suitable for sub-pathways.

Taking the top three ranked abnormal sub-pathways as examples, we extracted their sub-networks for the sub-pathway network, as shown in Fig. 2. The first one, abnormal sub-pathway for PI3K-Akt signaling pathway (ID: 4151_2), consisted of 21 nodes (7 signature lncRNAs, 10 interesting mRNAs and 4 genes) and 23 edges, such as TTTY15, DLEU2 and UBXN8. There were 8 interesting mRNAs, 4 signature lncRNAs, 4 genes and 23 edges in the sub-network for longterm potentiation (ID: 4720_1). The signature lncRNA DLEU2 connected more modes than the others, and thus, it was critical for this sub-pathway. As for the sub-network for pathways in cancer (ID: 5200_2), a total of 27 nodes and 37 edges were mapped on it, of which 17 interesting mRNAs, 8 signature lncRNAs and 2 genes, especially for UBXN8.

\section{Discussion}

Although numerous studies have concentrated on the relevance of lncRNAs to tumorigenesis as well as other diseases, the exact functions and activities of lncRNAs in PO progression remains unclear. Accurate molecular mechanisms underlying PO are controversial. For instance, Stuss et al considered that hormonal changes, which occurred throughout perimenopause and the immediate postmenopausal years, led to the accelerated $\mathrm{BMD}$ loss by stimulating the receptor activator of nuclear factor- $\kappa \beta$ (RANK) and its ligand (RANKL) production (26). To shed new light on the dispute of PO, in this study, we revealed the pathological mechanism of PO from the view point of lncRNAs.

Since the RNA competitive interaction can influence the various critical functions of disease, investigating LCRPs not only can gain insight into the potential mechanism but also help investigate the lncRNAs functional role in disease (12). Thus, we identified abnormal lncRNAs between PO and controls by the integration of IncRNA-mRNA expression profiles and pathway network topology. As IncRNAs and mRNAs were not worked on directly, we built their relations through the shared miRNAs from lncRNA-miRNA and miRNA-mRNA interactions, and obtained interesting lncRNA-mRNA pairs for PO. To enhance the feasibility and confidence of our study, we chose the significant KEGG pathways as graphs to link lncRNA to pathways, and consequently obtained LCRPs. It was revealed that signature lncRNAs combined with topology of LCRPs could efficiently give light on positioning of lncRNA-regulated sub-regions (12). Under this condition, abnormal sub-pathways between PO patients and controls were detected by the Wallenius approximation methods. A total of 14 abnormal sub-pathways were investigated, such as sub-pathways of PI3K-Akt signaling pathway and long-term potentiation. Besides, we uncovered several critical lncRNAs 


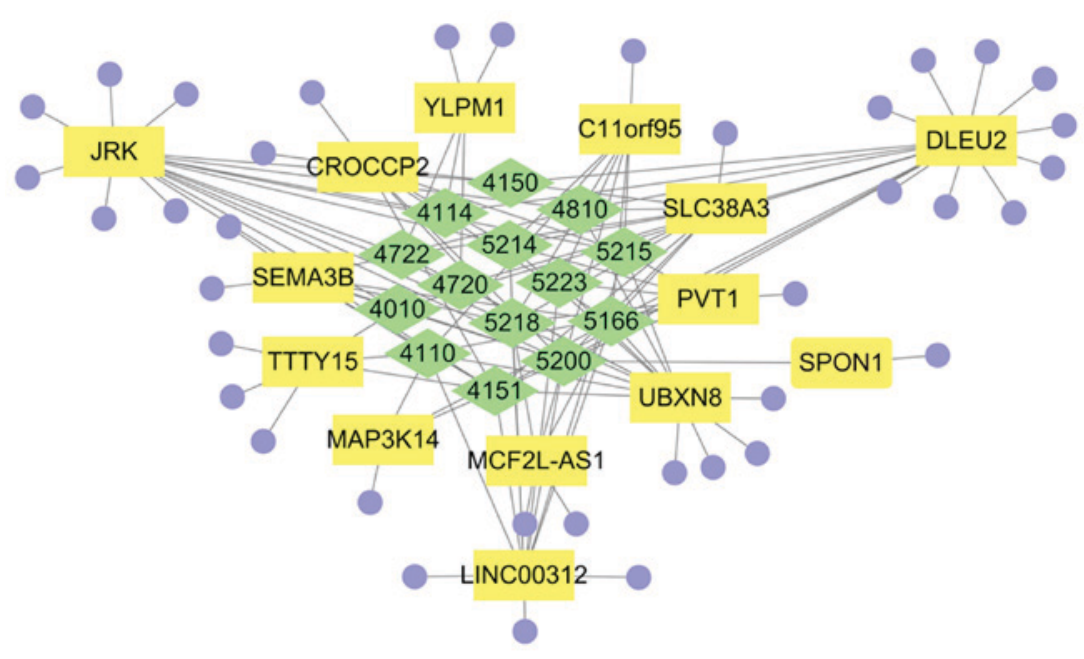

Figure 1. Network for abnormal sub-pathways. Circle and square nodes represent mRNAs and lncRNAs, respectively. While rhombus nodes referred to the ID of LCRPs corresponding to abnormal sub-pathways, and the edges stand for the connectivity of two nodes. IncRNA, long non-coding RNA; mRNA, messenger RNA; LCRPs, IncRNA competitively regulated pathways.

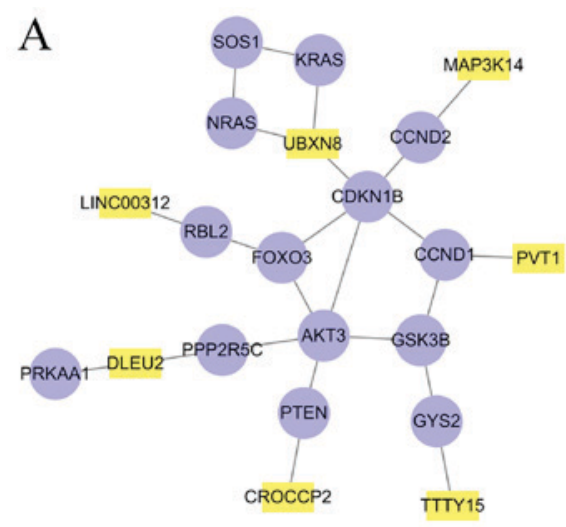

B
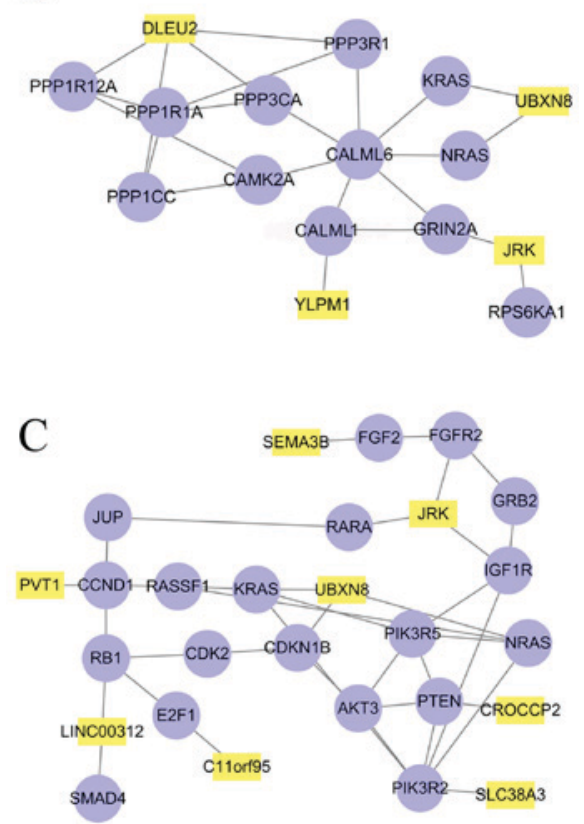

Figure 2. Network for abnormal sub-pathway. (A) PI3K-Akt signaling pathway, (B) long-term potentiation and (C) pathways in cancer. Circle and square nodes represented mRNAs and lncRNAs, respectively. The edges stand for the interactions of two nodes. IncRNA, long non-coding RNA; mRNA, messenger RNA. that regulated the important abnormal sub-pathways, for example, TTTY15, DLEU2 and UBXN8. The mRNAs in the sub-network of sub-pathway might be biomarkers for treatment and prevention of PO to some extent. On the basis of these results, the potential molecular mechanism of PO should be clarified.

Taking PI3K-Akt signaling pathway for an example, it is activated via multiple types of toxic insults or cellular stimuli and adjusts the fundamental functions of cells (i.e. proliferation, transcription, growth, translation, and survival) (27). Generally, the PI3K and AKT combined with mTOR pathways are deregulated in human cancer leading to genetic alterations in their components or upstream activation of cell-surface receptors (28). In this study, we also found that mTOR signaling pathway was a significant pathway for PO patients, which confirmed a previous study. In addition, Sabine et al revealed that deregulation of PI3K/AKT pathway genes contributed to the endocrine resistance of breast cancer (29), which indicated that this signaling pathway correlated with the endocrine changes in women. Thus, we might infer that the abnormal regulations of certain parts in PI3K-Akt signaling pathway caused the BMD loss in PO patients by affecting their endocrine changes.

In conclusion, we have successfully identified abnormal sub-pathways for PO patients compared with normal controls by integrating lncRNA related analysis and pathway topologies. The findings might shed new light on revealing potential molecular mechanism underlying PO, and provide several signature IncRNAs with significant functions in the progression of PO. Further studies should focus on validation of these signature lncRNAs.

\section{Acknowledgements}

Not applicable.

\section{Funding}

No funding was received. 


\section{Availability of data and materials}

The datasets used and/or analyzed during the present study are available from the corresponding author on reasonable request.

\section{Authors' contributions}

BGG conceived the study and drafted the manuscript. XXC acquired the data. BGG and XXC analyzed the data and revised the study. Both authors read and approved the final study.

\section{Ethics approval and consent to participate}

Not applicable.

\section{Patients consent for publication}

Not applicable.

\section{Competing interests}

The authors declare that they have no competing interests.

\section{References}

1. Watts NB, Bilezikian JP, Camacho PM, Greenspan SL, Harris ST, Hodgson SF, Kleerekoper M, Luckey MM, McClung MR, Pollack RP, et al: American Association of Clinical Endocrinologists Medical Guidelines for Clinical Practice for the diagnosis and treatment of postmenopausal osteoporosis. Endocr Pract 3: 1-37, 2010.

2. Unni S, Yao Y, Milne N, Gunning K, Curtis JR and LaFleur J: An evaluation of clinical risk factors for estimating fracture risk in postmenopausal osteoporosis using an electronic medical record database. Osteoporos Int 26: 581-587, 2015.

3. Reid IR, Bolland MJ and Grey A: Effects of vitamin D supplements on bone mineral density: A systematic review and meta-analysis. Lancet 383: 146-155, 2014.

4. Neer RM, Arnaud CD, Zanchetta JR, Prince R, Gaich GA Reginster JY, Hodsman AB, Eriksen EF, Ish-Shalom S, Genant HK, et al: Effect of parathyroid hormone (1-34) on fractures and bone mineral density in postmenopausal women with osteoporosis. N Engl J Med 344: 1434-1441, 2001.

5. Lips P: Epidemiology and predictors of fractures associated with osteoporosis. Am J Med 103: 3S-8S; discussion 8S-11S, 1997.

6. McClung MR, Grauer A, Boonen S, Bolognese MA, Brown JP, Diez-Perez A, Langdahl BL, Reginster JY, Zanchetta JR, Wasserman SM, et al: Romosozumab in postmenopausal women with low bone mineral density. N Engl J Med 370: 412-420, 2014.

7. Ebert MS and Sharp PA: Emerging roles for natural microRNA sponges. Curr Biol 20: R858-R861, 2010.

8. Tay Y, Rinn J and Pandolfi PP: The multilayered complexity of ceRNA crosstalk and competition. Nature 505: 344-352, 2014

9. Koyutürk M, Grama A and Szpankowski W: An efficient algorithm for detecting frequent subgraphs in biological networks. Bioinformatics 20 (Suppl 1): i200-i207, 2004.

10. Li C, Han J, Yao Q, Zou C, Xu Y, Zhang C, Shang D, Zhou L, Zou C, Sun Z, et al: Subpathway-GM: Identification of metabolic subpathways via joint power of interesting genes and metabolites and their topologies within pathways. Nucleic Acids Res 41: e101, 2013.

11. Li C, Li X, Miao Y, Wang Q, Jiang W, Xu C, Li J, Han J, Zhang F, Gong B, et al: SubpathwayMiner: A software package for flexible identification of pathways. Nucleic Acids Res 37: e131, 2009.
12. Shi X, Xu Y, Zhang C, Feng L, Sun Z, Han J, Su F, Zhang Y, Li C and Li X: Subpathway-LNCE: Identify dysfunctional subpathways competitively regulated by lncRNAs through integrating lncRNA-mRNA expression profile and pathway topologies. Oncotarget 7: 69857-69870, 2016.

13. Yang JH, Li JH, Shao P, Zhou H, Chen YQ and Qu LH: starBase: A database for exploring microRNA-mRNA interaction maps from Argonaute CLIP-Seq and Degradome-Seq data. Nucleic Acids Res 39 (suppl_1): D202-D209, 2011.

14. Xiao F, Zuo Z, Cai G, Kang S, Gao X and Li T: miRecords: An integrated resource for microRNA-target interactions. Nucleic Acids Res 37 (Database): D105-D110, 2009.

15. Skala M: Hypergeometric tail inequalities: Ending the insanity. Statistics, eprint arXiv:1311.5939, 2013. https://arxiv.org/ pdf/1311.5939.pdf.

16. Niwattanakul S, Singthongchai J, Naenudorn E and Wanapu S: Using of Jaccard Coefficient for Keywords Similarity. Lecture Notes in Engineering \& Computer Science, 2013. In: International MultiConference of Engineers and Computer Scientists 2013 Vol I. Hong Kong, pp380-384, 20013.

17. Irizarry RA, Bolstad BM, Collin F, Cope LM, Hobbs B and Speed TP: Summaries of Affymetrix GeneChip probe level data. Nucleic Acids Res 31: e15, 2003.

18. Bolstad BM, Irizarry RA, Astrand M and Speed TP: A comparison of normalization methods for high density oligonucleotide array data based on variance and bias. Bioinformatics 19: 185-193, 2003.

19. Nahler G: Pearson correlation coefficient. In: Dictionary of Pharmaceutical Medicine. Springer, Vienna, pp132, 2009.

20. Best DJ and Roberts DE: Algorithm AS 89: The Upper Tail Probabilities of Spearman's Rho. J R Stat Soc [Ser A] 24: 377-379, 1975.

21. Whitlock MC: Combining probability from independent tests: The weighted Z-method is superior to Fisher's approach. J Evol Biol 18: 1368-1373, 2005.

22. Routledge R: Fisher's Exact Test. In: Encyclopedia of Biostatistics. Armitage P and Colton T (eds). John Wiley \& Sons, Ltd., New York, NY, 2005.

23. Benjamini Y, Drai D, Elmer G, Kafkafi $\mathrm{N}$ and Golani I: Controlling the false discovery rate in behavior genetics research. Behav Brain Res 125: 279-284, 2001.

24. Fog A: Calculation methods for Wallenius' noncentral hypergeometric distribution. Commun Stat Simul Comput 37: 258-273, 2008.

25. Epstein MP, Duncan R, Jiang Y, Conneely KN, Allen AS and Satten GA: A permutation procedure to correct for confounders in case-control studies, including tests of rare variation. Am J Hum Genet 91: 215-223, 2012.

26. Stuss M, Rieske P, Cegłowska A, Stêpień-Kłos W, Liberski PP, Brzeziańska E and Sewerynek E: Assessment of OPG/RANK/RANKL gene expression levels in peripheral blood mononuclear cells (PBMC) after treatment with strontium ranelate and ibandronate in patients with postmenopausal osteoporosis. J Clin Endocrinol Metab 98: E1007-E1011, 2013.

27. Martini M, De Santis MC, Braccini L, Gulluni F and Hirsch E: PI3K/AKT signaling pathway and cancer: An updated review. Ann Med 46: 372-383, 2014.

28. De Luca A, Maiello MR, D'Alessio A, Pergameno $M$ and Normanno N: The RAS/RAF/MEK/ERK and the PI3K/AKT signalling pathways: Role in cancer pathogenesis and implications for therapeutic approaches. Expert Opin Ther Targets 16 (Suppl 2): S17-S27, 2012.

29. Sabine VS, Crozier C, Brookes CL, Drake C, Piper T, van de Velde CJ, Hasenburg A, Kieback DG, Markopoulos C, Dirix L, et al: Mutational analysis of PI3K/AKT signaling pathway in tamoxifen exemestane adjuvant multinational pathology study. J Clin Oncol 32: 2951-2958, 2014.

This work is licensed under a Creative Commons Attribution-NonCommercial-NoDerivatives 4.0 International (CC BY-NC-ND 4.0) License. 